\title{
Contact points
}

Recorded announcement of latest RPI

C. 02075335866

国 rpi@ons.gsi.gov.uk

Labour Market Statistics Helpline

C. 02075336094

(abour.market@ons.gsi.gov.uk

\section{For statistical information on}

Average Earnings Index (monthly)

(C) 01633819024

Claimant count

C. 02075336094

Consumer Prices Index

C) 02075335874

Earnings

Annual Survey of Hours and Earnings

C. 01633819024

Basic wage rates and hours for manual workers with a collective agreement

C 01633819008

Low-paid workers

C 01633819024

(凶) lowpay@ons.gsi.gov.uk

Labour Force Survey

C. 02075336094

() labour.market@ons.gsi.gov.uk

Economic activity and inactivity

C) 02075336094

Employment

Labour Force Survey

(C) 02075336094

(a) labour.market@ons.gsi.gov.uk

Employee jobs by industry

C) 01633812318

Total workforce hours worked per week

(C) 01633812766

国 productivity@ons.gsi.gov.uk

Workforce jobs series -

short-term estimates

C 01633812318

() workforce.jobs@ons.gsi.gov.uk
Earnings Customer Helpline

C 01633819024

(ه) earnings@ons.gsi.gov.uk

National Statistics Customer Contact Centre

C 08456013034

(2) info@statistics.gsi.gov.uk
Skills and Education Network

C 02476823439

(国 senet@isc.gov.uk

DfES Public Enquiry Unit

C 08700002288
Labour costs

C 01633819024

Labour disputes

C 01633819205

Labour Force Survey

C 02075336094

(지 labour.market@ons.gsi.gov.uk

Labour Force Survey Data Service

C 01633655732

ه Ifs.dataservice@ons.gsi.gov.uk

New Deal

C 01142098228

Productivity and unit wage costs

C 01633812766

Public sector employment

General enquiries

C 02075336178

Source and methodology enquiries

C 01633812362

Qualifications (DfES)

C 08700002288

Redundancy statistics

C 02075336094

Retail Prices Index

C 02075335874

직i@ons.gsi.gov.uk

Skills (DfES)

( 01142594407

Skill needs surveys and research into skill shortages

C 01142594407

Small firms (DTI)

Small Business Service (SBS)

C 01142794439

\section{Subregional estimates}

C 01633812038

\section{Annual employment statistics \\ (凶annual.employment.figures@ons.gsi. gov.uk}

Annual Population Survey, local area statistics

C) 02075336130

LFS Subnational Data Service

C) 02075336135

国 snds@ons.gsi.gov.uk

Trade unions (DTI)

Employment relations

C) 02072155934

Training

Adult learning - work-based training (DWP)

C 01142098236

Employer-provided training (DfES)

C 01142594407

Travel-to-Work Areas

Composition and review

C 02075336114

Unemployment

C. 02075336094

Vacancies

Vacancy Survey:

total stocks of vacancies

C 02075336162 